\title{
Effect of Ni doping on ferroelectric and dielectric properties of strontium barium niobate crystals
}

\author{
K. Matyjasek $\cdot$ K. Wolska $\cdot$ S.M. Kaczmarek • \\ J. Subocz $\cdot$ L. Ivleva
}

Received: 28 February 2011 / Revised version: 1 September 2011 / Published online: 11 October 2011

(C) The Author(s) 2011. This article is published with open access at Springerlink.com

\begin{abstract}
The influence of $\mathrm{Ni}$ doping on the ferroelectric and dielectric properties have been examined in $\mathrm{Sr}_{0.61} \mathrm{Ba}_{0.39}$ $\mathrm{Nb}_{2} \mathrm{O}_{6}$ (SBN:61) relaxor crystals. The dopants introduced into $\mathrm{SBN}$ :61 crystals promote the switching process by reducing the value of threshold nucleation field, and thus coercive field. We present real-time studies of domain nucleation and growth processes in doped SBN:61 by the nematic liquid crystal (NLC) decoration technique. The broad phase transition and low-frequency dielectric dispersion that are exhibited by doped SBN:61 samples have a strong link to the configuration of the ferroelectrics microdomains, which in turn is strongly determined by $\mathrm{Ni}$ ions concentration.
\end{abstract}

\section{Introduction}

The relaxor ferroelectrics, strontium barium niobates, $\mathrm{Sr}_{0.61} \mathrm{Ba}_{0.39} \mathrm{Nb}_{2} \mathrm{O}_{6}$ (SBN:61) have received a great interest in their applications in optoelectronics, due to their large electro-optical and relatively high nonlinear optical coefficients [1]. In most of the relevant physical effects used in the applications, such as optical frequency conversion and high

K. Matyjasek $(\bowtie) \cdot$ K. Wolska $\cdot$ S.M. Kaczmarek

Institute of Physics, Faculty of Mechanical Engineering and Mechatronics, West Pomeranian University of Technology,

Al. Piastow 48, 70-310 Szczecin, Poland

e-mail:km@zut.edu.pl

J. Subocz

Faculty of the Electrical Engineering, West Pomeranian University of Technology, Sikorskiego St. 37, 70-313 Szczecin, Poland

L. Ivleva

Institute of General Physics, Russian Academy of Sciences,

Vavilov St. 38, 119991 Moscow, Russia density optical data storage, the specific domain patterns are required. Therefore, disclosing the nature of the switching mechanism in external electric field remains of particular scientific and technological interest $[2,3]$. The main features of relaxors include a very broad and frequencydependent dielectric anomaly with the high dielectric permittivity values [4]. The data reveal that the maximum of dielectric permittivity for SBN shifts toward higher temperatures with increasing frequency. Despite experimental investigations of dielectric relaxation, the microscopic origin of unusual physical properties of relaxors still remains a point of discussion [5]. The open tungsten bronze structure of SBN allows various possibilities to accommodate different doping ions, taking into account that only five out of six available positions of $\mathrm{Sr}^{2+}$ and $\mathrm{Ba}^{2+}$ host cations are occupied [6]. In such crystals, the origin of the relaxor behavior can be attributed to the development of a quenched random field associated with the compositional/structural disorder [7]. Doping SBN with rare-earth or alkali ions improves the properties via a significant increase of the electrooptical and piezoelectric coefficients [8-10]. The introduction of rare-earth metals is accompanied by a substantial decrease in the phase transition temperature and brings about a considerable change in the dielectric permittivity, over a wide range of temperatures [11, 12]. It was also established that the doping with rare-earth metals results in a "retardation" of the switching process [12]. On the other hand, the alkali $\left(\mathrm{Li}_{0.5} \mathrm{Na}_{0.5}\right)$ doping SBN results in increasing the phase transition temperature along with decreasing the maximum dielectric constant [13].

In order to understand this anomalous behavior in different doped SBN crystals, we examined the ferroelectric and dielectric properties in SBN:61 crystals doped with a different amount of transition metal impurity of $\mathrm{Ni}$ additives. It was reported that $\mathrm{Ni}$ doping in SBN:61 results in a re- 
markable change in the piezoelectric and dielectric coefficients and leads to a substantial decrease in the phase transition temperature $[8,9]$. To the best of our knowledge, there is no report on the ferroelectric properties and the domain structure in Ni-substituted SBN:61 single crystals. There are very limited works devoted to study of the influence of doping on ferroelectric properties, especially the polarization switching mechanism. Until recently, high-resolution studies of SBN crystals were limited to piezoresponse force microscopy (PFM) techniques, providing the visualization of the domain structure at the nanoscale [14-17]. In the PFM experiment conditions, the electric field is strongly localized and inhomogeneous. A microscale domain state in SBN crystals was observed by electrooptic imaging microscope [18, 19] and the NLC method [20, 21]. The NLC method involves averaging over macroscopic scale and this enables as to correlate the domain structure dynamics with the macroscopic characterization techniques; switching current, hysteresis loop, and dielectric permittivity measurements. Based on direct observation of the domain structure evolution during the slow polarization reversal in low electric fields, together with electrical measurements in high fields, valuable information concerning the mechanism of the polarization switching in Ni doped SBN:61 crystal samples can be obtained. This paper reports that ferroelectricity can be substantially enhanced in Ni doped SBN:61 crystal samples. Particularly, the doped samples showed larger remnant polarization $\left(P_{\mathrm{r}}\right)$ and a small coercive field $\left(E_{\mathrm{c}}\right)$ in comparison with the undoped ones, at a given measuring ac electric field. The authors also present experimental data, which may shed more light, on the physical nature of dielectric response in the impurity induced dielectric anomalies.

\section{Experimental details}

SBN crystal exhibits only $180^{\circ}$ domains because the paraelectric phase has a tetragonal symmetry $(4 / \mathrm{mmm})$, and the order parameter in the ferroelectric phase $(4 \mathrm{~mm})$ occurs along [001] direction [22]. Details of the growing procedure, by a modified Stepanov method, are presented elsewhere [23]. Doping was performed by adding $\mathrm{Ni}_{2} \mathrm{O}_{3}$ (0.01 and 0.5 wt.\%) in the melt. Plateled-shaped samples were cut perpendicularly to the [001] direction and polished to optical quality. Before all measurements, the samples were annealed with high temperature to remove memory effects. Hysteresis loops (D-E dependence) were recorded using a modified Sawyer-Tower circuit by using an ac field of $50 \mathrm{~Hz}$. Switching current transients were measured by use a wave-form function generator, a small standard resistor and digital oscilloscope. A set of square pulses (of $10 \mathrm{~ms}$ duration) was applied by combining two positive and two negative square pulses in series. A true switching current transient was then obtained by subtracting the nonswitching current from the switching one, for a given value of an electric field. Then the switching current data were integrated in order to obtain the temporal dependence of the switched polarization. The direct observation of the domain structure evolution in low electric field and electrical measurements in high fields were carried out at room temperature, i.e., in the ferroelectric phase. Dielectric permittivity dependence on temperature and frequency was measured by an HP 4284A LCR meter. The electric measurements were carried out with an air-drying silver paste as electrodes.

The NLC method enables one to observe directly the single domains (up to $1 \mu \mathrm{m}$ in size) and the entire surface of the crystal sample, during polarization reversal. To observe optically indistinguishable $180^{\circ}$ domain walls, the NLC mixture of p-methoxybenzylidene-p-n-butylaniline (MBBA) and pethoxybenzylidene-p-n-butylaniline (EBBA) was used. The static domain structure gave no contrast. For dynamic observation in an electric field, a crystal plate with a thin NLC layer, on its upper and lower surface, was sandwiched by two glass plates with a tin oxide coating as transparent electrodes. The domains were observed by a polarizing microscope and recorded with a digital camera. In the region, where reorientation of polarization direction takes place, switching current flows followed by a decrease in the impedance of the ferroelectric crystal; hence, the major part of the applied voltage falls on the NLC layer. Thus, the regions where reorientation of domains still occurs look somewhat darker, as in these regions a certain electrohydrodynamic instability, particularly dynamic scattering, takes place [24]. Some limitations of this method are connected with a slow response time of NLC molecules (of the order of several ms). Moreover, the voltage is applied to the sandwich structure consisting of ferroelectric sample and NLC layers (the thickness of NLC layer of about several $\mu \mathrm{m})$ with a specific resistance of the order of $10^{2} \Omega \mathrm{m}$. Thus, it is difficult to estimate the real magnitude of the voltage applied to the ferroelectric sample, especially during the switching process. The results of the reported works show a strong influence of the interface (electrode and ferroelectric surface) conductivity on the condition for compensation of depolarization field, produced by bound charges, and thus on the kinetics of the polarization reversal [25]. Thus, the switching kinetics is determined by the conductivity of the NLC layer. Therefore, the visualization of the domain structure by NLC method can only be used in checking the domain distribution and growth during the switching process, which obviously reflects internal disorder of the crystal. 
Fig. 1 Family of D-E hysteresis loops obtained at room temperature by applying ac-electric fields of $50 \mathrm{~Hz}$ :

(1) undoped SBN:61 sample,

(2) $\mathrm{Ni}(0.01 \mathrm{wt} . \%)$ and

(3) $\mathrm{Ni}(0.5$ wt.\%) doped SBN sample. Remnant polarization

$P_{\mathrm{r}}$ coercive field $E_{\mathrm{c}}$ versus

electric field amplitude (a)
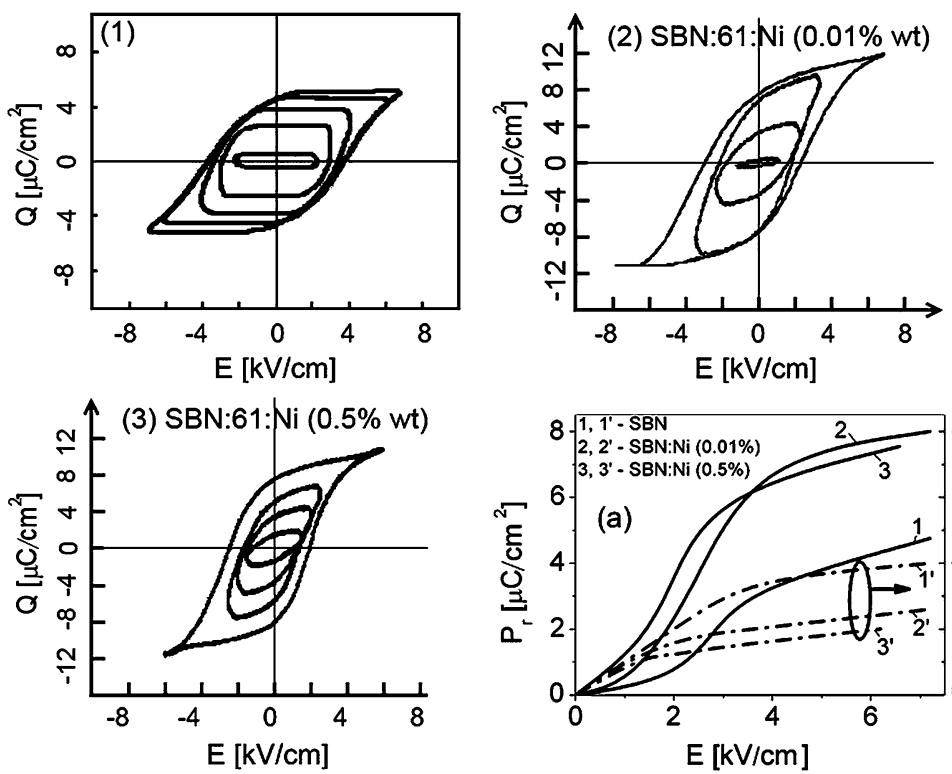

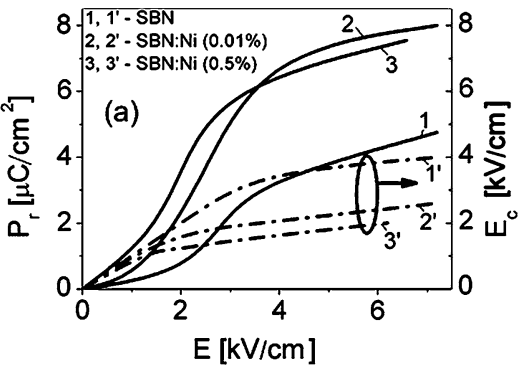

\section{Results}

\subsection{Hysteresis loop measurements}

Figure 1 shows typical (D-E) hysteresis loops, obtained at room temperature for examined crystal samples. The improvement mechanism of switching has been observed in doped crystal samples. Particularly, the coercive field $\left(E_{\mathrm{c}}\right)$ decreases and remnant polarization $\left(P_{\mathrm{r}}\right)$ increases in both doped crystal samples. The variation of the remnant polarization and the coercive field with the applied field for different Ni doped SBN:61 samples is shown in Fig. 1(a). In the case of doped samples, the coercive field decreases by about twice in comparison with the undoped ones, at the same applied ac electric field. Since there is no obvious leakage current contribution in the hysteresis loops, the remnant polarization of these hysteresis loops arises essentially from the ferroelectric domains. The examined crystal samples have no precisely defined $E_{\mathrm{c}}$, which increases steadily with the increase of the electric field amplitude. These results suggest that in these crystals, even in high electric fields, there are frozen regions that do not participate in the switching polarization and/or that a large contribution to the polarization is due to sidewise domain wall motion, which is a very slow mechanism. The hysteresis loop (H-L) measurements investigate collective switching process of all domains under the electrode. Local microscopic observations can reveal more about nucleation and domain growth during the switching process.

\subsection{Domain structure dynamics}

Optical techniques are much more valuable in obtaining spatiotemporal information about the polarization switch- ing and are useful completion to the usual H-L measurements. The domain ordering behavior in ferroelectric crystal samples is influenced by their (i) compositional inhomogeneity and (ii) defect microstructure. Formation of the microscale domains, in the monodomain crystal sample, is possible if the amplitude of the dc electric field exceeds some threshold value, at a given site of the crystal. It has been recently reported that even nominally pure SBN:61 crystal samples are inhomogeneous; some regions of the crystal sample are switched very fast (with large density of nucleated domains) and there are regions where nucleation process is suppressed [26]. The fact that the similar picture of the delineated domains has been observed for positive and negative electric field demonstrates the presence of a frozen polarization state, possibly due to locally accumulated defects. There are large quantitative differences in the switching parameters of different undoped SBN:61 crystal samples. Within particular crystals, more than a $50 \%$ variation in coercive field have been measured, at the same applied ac electric field.

Figure 2 illustrates the domain pattern evolution observed in more homogeneous fragment (with spatially uniform position of nucleated domains) of the undoped sample during switching in $E=2.8 \mathrm{kV} \mathrm{cm}^{-1}$. Before applying $E$, the crystal was in a single domain state created by external field of opposite orientation The electric field of $2.8 \mathrm{kV} \mathrm{cm}^{-1}$ is high enough to reverse the polarization direction in the most switchable volume of the crystal sample that was reversed within the time of several seconds. However, nearly onefifth of the crystal under the electrode area was not reversed in this electric field. In the regions with a frozen polarization state, the domain nucleation process was distinguished after application of a higher field, $E>3.6 \mathrm{kV} \mathrm{cm}^{-1}$. This may 

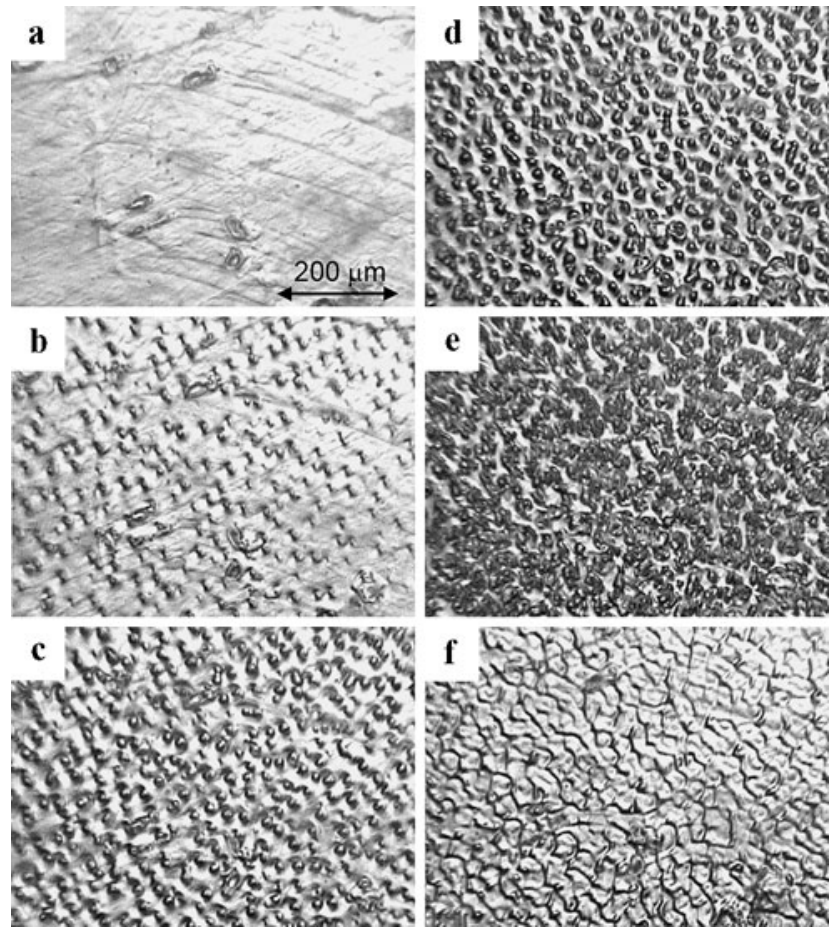

Fig. 2 Selected video frames in video recording of domain pattern evolution, observed on the (001) plane of the undoped SBN:61 sample during the switching in electric field of $2.8 \mathrm{kV} \mathrm{cm}^{-1}$. (a) Initial single domain state. Time from the moment of applying $E$ in [s]: (b) 0.24 ; (c) 0.8 ; (d) 2 ; (e) 3 ; (f) 5 ; the domain walls were clearly distinguished after application of the electric field of $3.2 \mathrm{kV} \mathrm{cm}^{-1}$ The switching process was completed within the time of $7.5 \mathrm{~s}$ in the observed surface area $\sim 0.4 \mathrm{~mm}^{2}$

explain the fact that the undoped crystal sample has no precisely defined coercive field, which increases steadily with the increase of the electric field. The dark areas shown in Fig. 2 correspond to areas which actually reversed their polarization direction and clear areas correspond to the regions where the switching process has not started (initial state in Fig. 2(a)) or has already been completed (see Fig. 2(f)). The sidewise movement of the domain walls is strongly perturbed by the random field environment, related to the relaxor properties of the SBN crystals, and resulting domain wall pinning effect [7]. A marked slowing down of the domain wall velocities was observed in the final stage of the switching process, where low-mobility regions (clear areas in Fig. 2(e)) restrict the coalescence of domains. It is reflected in a poor orientation contrast of the NLC above the slowly moving domain walls. In order to reveal the domain boundaries that exist just before coalescence of domains (dark color lines seen in Fig. 2(f)), a slightly higher electric field $E=3.2 \mathrm{kV} \mathrm{cm}^{-1}$ was applied. Polarization develops in two distinct steps; it increases rapidly (dominated by domain nucleation) for the first few tenths of seconds, immediately after applying $E$, and then slowly (for a few seconds) by sidewise domain wall motion toward the saturation state. The first stage has a threshold nucleation field and
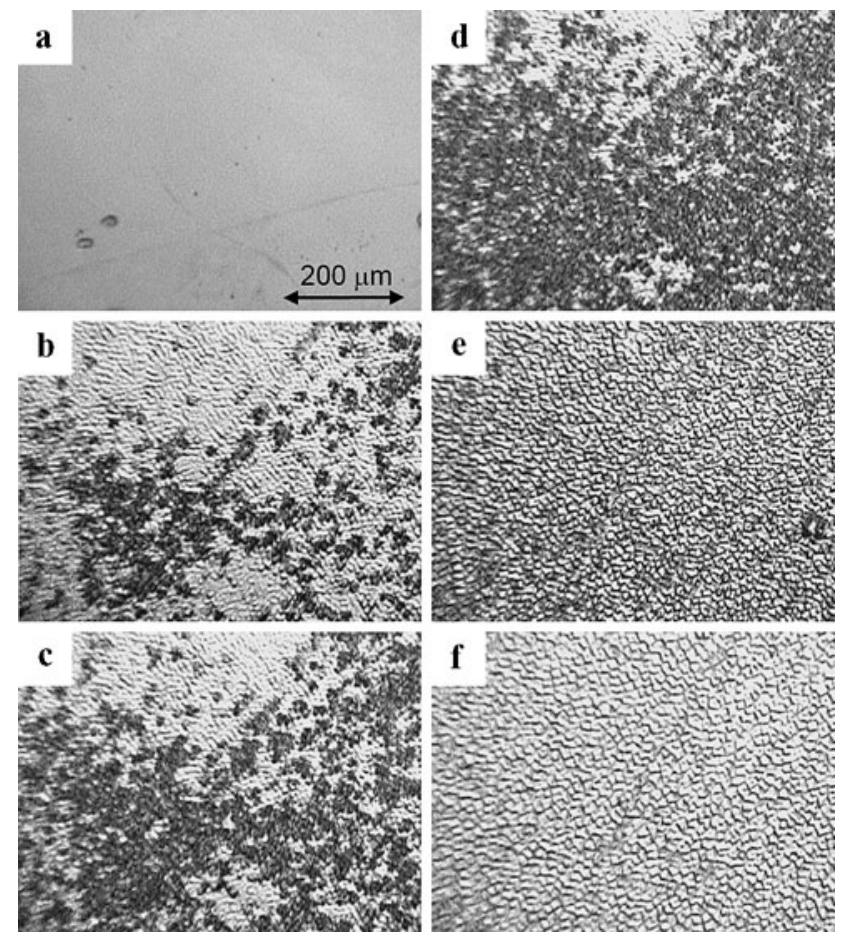

Fig. 3 Domain pattern evolution in Ni (0.01 wt.\%) doped SBN:61 sample. (a) Initial single domain state. Time from the moment of applying $E=2.5 \mathrm{kV} \mathrm{cm}^{-1}$ in [s]: (b) $0.12 ;$ (c) 0,16 ; (d) 0,$2 ;$ (e) 2 ; (f) 3 . The switching process was completed within the time of $4.6 \mathrm{~s}$ in the observed surface area $\sim 0.4 \mathrm{~mm}^{2}$

corresponds to the forward growth of domains. The threshold nucleation field, $E_{\mathrm{th}}$, equal $2.4 \mathrm{kV} \mathrm{cm}^{-1}$ for the undoped sample, is chosen as the external field that must be applied to the crystal sample so as to initiate domain nucleation and growth process from a single-domain state. The term "nucleation" is used to describe the emergence of new, sharp visible, antiparallel domains within the original single domain state, as they appear in the video images. However, it is an open question whether the initial domain state is single-domain or contains the nanoscale residual domains, not resolved by NLC method. Nanodomains have been directly observed in Ce-doped SBN:61 using high-resolution PFM techniques [14].

For slightly doped SBN:61 (0.01 wt.\% Ni) crystal sample, as is seen in Fig. 3, the process of domain nucleation is spatially more inhomogeneous, initiated at static impurities or defect sites that are fixed in space. The nucleation process takes place nearly during the whole polarization reversal process, at an electric field of $2.5 \mathrm{kV} \mathrm{cm}^{-1}$ $\left(E_{\mathrm{th}}=2.0 \mathrm{kV} \mathrm{cm}^{-1}\right)$. This field is high enough to complete the microscale domain switching in the entire switchable volume of the crystal sample, because application of higher electric fields ( $\left.E>2 E_{\mathrm{c}}\right)$ do not induce any domain switching. The sites in which intensive nucleation takes place are seen as "black" regions. The specific mechanism driving the nucleation results from the local structural irregularities, and 

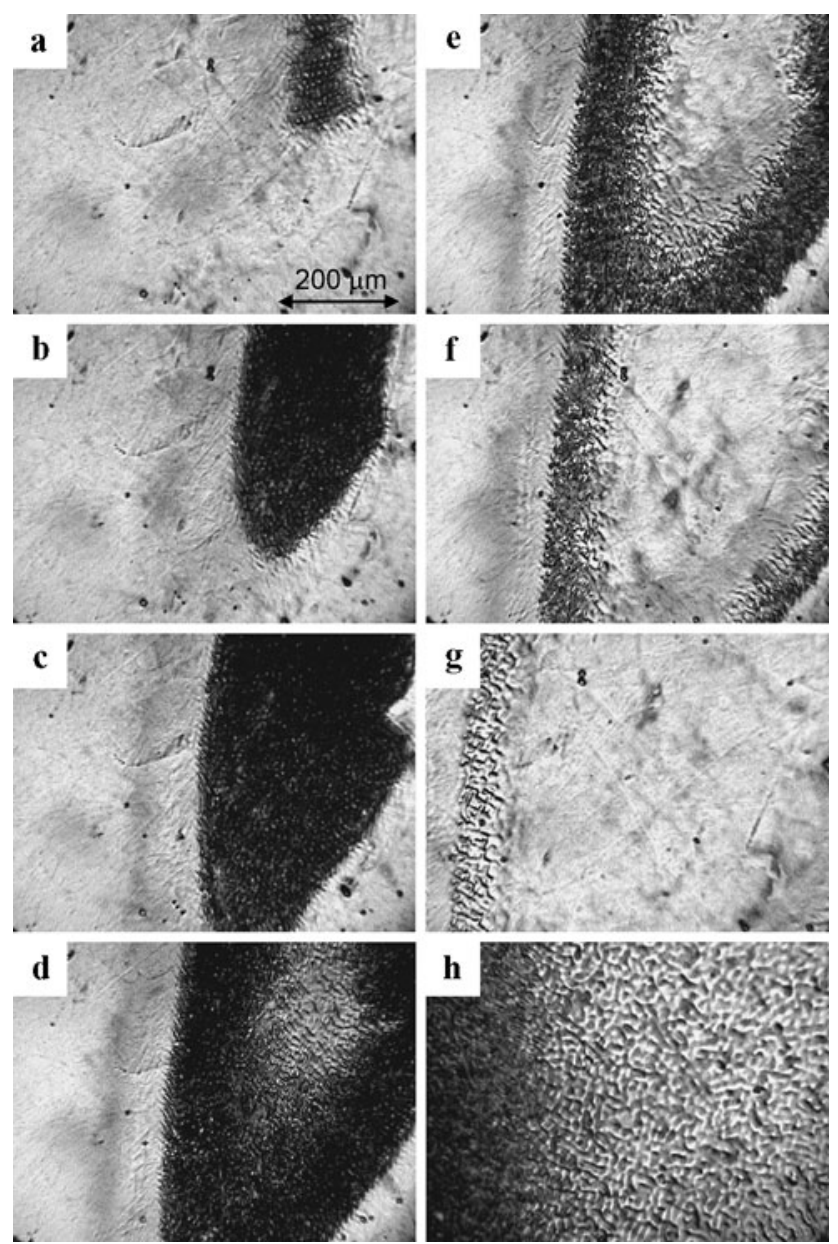

Fig. 4 Domain pattern evolution in Ni (0.5 wt.\%) doped SBN:61 sample. Time from the moment of applying $E=1.1 \mathrm{kV} \mathrm{cm}^{-1}$ in [s]: (a) 0.04 ; (b) $0.24 ;$ (c) 0,6 ; (d) 1.2 ; (e) 1.6 ; (f) 2.0 ; (g) 3.6 ; (h) the motion of the domain walls was distinguished following the application of the electric field of $2 \mathrm{kV} \mathrm{cm}^{-1}$

could be interpreted in terms of a wide distribution of activation energy for nucleation. In sites where the nucleation process is suppressed, the macroscopically visible domain walls arise with lower wall velocity (reflected in a poor contrast of NLC above these domain walls). One should expect that the appearance of domain nuclei takes place in the bulk of the crystal; they collapse forming macroscopically visible domain walls. It should be emphasized that the thickness of the "visible" walls are not real physical thickness of the domain walls, which are usually of the order of a few lattice constants. The nucleated domains do not grow continuously, as local structure irregularities provide strong pinning sites at which domain walls remain attached, forming a maze type domain pattern seen in the final stage of the polarization reversal process [Fig. 3(e)-(f)].

The heavy doping deteriorates the crystal homogeneity as is evidenced by spatially inhomogeneous nucleation process. Figure 4 shows the evolution of the domain structure in the highly doped SBN:61 (0.5 wt.\% Ni) crystal sample in the electric field of $1.1 \mathrm{kV} \mathrm{cm}^{-1}\left(E_{\mathrm{th}}=0.8 \mathrm{kV} \mathrm{cm}^{-1}\right)$. There are regions (shown in black), where intensive domain nucleation takes place, at the initial stage of the polarization reversal process [Fig. 4(a)-(c)]. The density of the nucleated domains is so high that they cannot be distinguished at this magnification. In the regions where the nucleation is suppressed, the switching continues to occur through the formation of new domains adjacent to the moving domain front previously formed [Fig. 4(e)-(g)]. This is a clear manifestation of the correlated nucleation effect, discussed in [27]. In highly defected crystal samples, the sidewise movement of the domain walls is strongly nonuniform, presumably due to domain interaction with microdefects. We calculated the domain wall speed, by the estimation the changes in the domain wall position versus time, from the moment when it propagated without interference from nearby nucleation centers. A marked slowing down of the domain wall velocities was observed during its propagation. The fluctuations of the domain wall velocity by nearly three orders of magnitude are indicative of a broad distribution of the domain wall mobility. The pronounced slowing down of the domain wall motion is seen in the final stage of the switching process, just before coalescence of domains. It is reflected in a poor contrast of NLC above the slowly moving domain walls, as is clearly seen in the region behind the domain wall front propagation (Fig. 4(f), (g)). In such regions, the sidewise motion of domain walls is hindered and requires the application of higher fields. The variation of the domain structure becomes noticeable (Fig. 4(h)) following the application of a higher electric field $E>2 \mathrm{kV} \mathrm{cm}^{-1}$ (of the order of $E_{\mathrm{c}}$ ) and such field is sufficient to complete the microscale domain switching in the entire volume of the crystal sample. We observed the following features as the electric field strength was increased. The domains nucleate sooner and in greater number per unit area, and the region with the intensive domain nucleation becomes larger. The inhomogeneous distribution of the domain nuclei can reflect the nonuniform defect structure distribution within the crystal sample. These observations suggest the presence of a frozen polarization component (in the regions where nucleation process is suppressed) or built-in field which favors one direction of the spontaneous polarization. The fact that this specific domain nucleation process was observed in both positive and negative polarization state demonstrates the presence of a frozen polarization state in the certain regions of the examined crystal sample. It is found that the higher electric fields, $E>2 \mathrm{kV} \mathrm{cm}^{-1}$, are sufficient to overcome internal fields that stabilize the domain structure. In the higher fields, the domain nucleation occurs immediately after the field application, and is observed on the whole surface area of the examined crystal sample. In consequence, in large external fields, the values of $P_{\mathrm{r}}$ and $E_{\mathrm{c}}$ are practically the same (Fig. 1(a)) in both Ni doped crystal samples. 


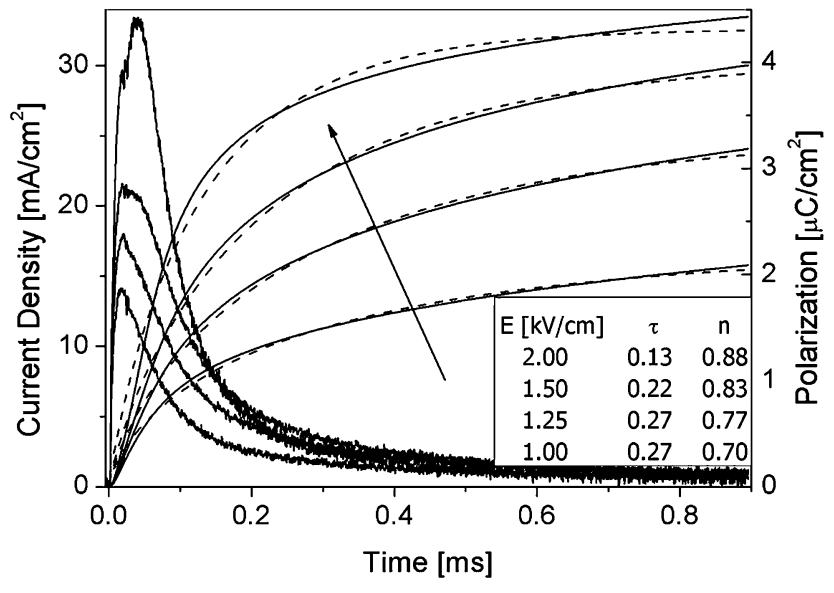

Fig. 5 Switching currents and switched polarization versus time for highly $\mathrm{Ni}(0.5 \mathrm{wt} . \%)$ doped SBN:61 sample

\subsection{Switching current measurements}

More comprehensive information about the fast switching process can be obtained from switching current curves, representing the reverse polarization as a function of the pulse duration for a constant electric field. Figure 5 presents the time dependence of the switched polarization obtained after integration of the switching curves for several amplitudes of external electric fields for SBN:61 (0.5 wt.\% Ni) crystal sample. In normal ferroelectrics, as the electric field increases, the switching curves shift to shorter times and the domain switching time is known to be of the order of microseconds, when external electric field approaches the $E_{\mathrm{c}}$. In relaxor SBN crystals, the switched polarization increases with the electric field strength, but does not saturate. The observed "partial" freezing of polarization may confirm the fact that there are slowly switching regions that do not contribute to the switching current signals, even in $E>E_{\mathrm{c}}$. This should be related to the pinning effect of the domain walls, resulting from a disordered structure of SBN crystals. The electric field of $2.0 \mathrm{kV} \mathrm{cm}^{-1}$ (the upper limit set in this experiment) is not sufficient to obtain complete reversal of polarization in the $10 \mathrm{~ms}$ pulse duration. The value of polarization found for $E=2 \mathrm{kV} \mathrm{cm}^{-1}$ is $\sim 4.8 \mu \mathrm{C} \mathrm{cm}^{-2}$. By comparison, the polarization obtained from $\mathrm{H}-\mathrm{L}$, at the same amplitude and pulse width of the acfield, is $\sim 4 \mu \mathrm{Ccm}^{-2}$. Thus, similarly as is observed in usual homogeneous ferroelectrics, the polarization reversal occurs more effectively under a dc field than under an ac field, at the some pulse amplitude. Two stages of the polarization relaxation can be distinguished. The first one occurs very quickly within the time range of tenths of milliseconds, and is accompanied mainly by domain nucleation. The instantaneous growth of residual domains may also lead to immediate jump-like increase of the current, just after applying $E$. The final stage of the switching process, representing a remarkable slowing down, is dominated by sidewise domain wall motion. The relaxation of the polarization during the switching process is well described by a Kohlrausch, Williams, and Watts (KWW) stretched exponential function $P(t)=P_{0}\left[1-\exp \left(-(t / \tau)^{n}\right)\right]$ with $0<n<1$, widely used in dielectric relaxation studies [28]. The fits are represented by the broken lines in Fig. 5. It is interesting to note that the KWW function described the switching kinetics in undoped SBN:61 [26] as well as relaxing domains on the nanoscale, with PFM imaging of the domain configuration in SNB:61 doped with cerium [14].

\subsection{Dielectric measurements}

Figure $6(\mathrm{a}-\mathrm{c})$ presents the dielectric characteristics of the examined crystal samples as a function of temperature at various frequencies. The dielectric constant, which implies the charge storage, has a strong dispersion in the lowfrequency region. For all the samples the maximum of dielectric constant decreased as the frequency increased. The values of $T_{\mathrm{m}}$ (temperature characterizing dielectric constant maximum) for slightly doped sample $(0.01 \mathrm{wt} . \% \mathrm{Ni})$ are nearly the same as in undoped SBN:61 $(\sim 353 \mathrm{~K}$ at $1 \mathrm{kHz})$. At a higher concentration of $\mathrm{Ni}(0.5 \mathrm{wt} . \%)$, the doped sample exhibits a very broad and frequency-dependent dielectric anomaly with low dielectric constant values, as seen in Fig. 6(c). Figure 6(d) shows the dielectric loss $(\tan \delta)$ as a function of temperature at frequency of $1 \mathrm{kHz}$. The dielectric loss which is dominated by the conduction of the mobile charges is reduced nearly twice in the doped crystal samples in comparison with the undoped one. Generally, the dielectric loss contribution for the polar substances comes from dipole losses and electric conduction losses [29]. The dipole losses increased up to a temperature close to the $T_{\mathrm{m}}$ and then decreased for undoped and slightly doped crystal samples (curve 1 and 2). The electric conduction losses increase with temperature; this effect is pronounced for highly doped SBN:61 (curve 3). The considerably high dielectric constant and relatively small dissipation factor could be obtained for the sample with a small $(0.01 \mathrm{wt} . \%) \mathrm{Ni}$ concentration.

\section{Discussion}

The domains in ferroelectric crystals are well known to have considerable influence on the value of their complex permittivity and related quantities $[29,30]$. It is reported that domain walls contribute to dielectric permittivity proportionally to their total surface [30]. One can presume that a weak measuring ac field in dielectric measurements did not affect the domain configuration but creates nuclei on the domain walls. Comparing the images in Figs. 2 and 3, one can observe that the density of the domain walls becomes larger in a slightly doped crystal sample (SBN:61 (0.01 wt.\% Ni) in 
Fig. 6 Temperature

dependences of dielectric

constants measured at different

frequencies for: (a) undoped

SBN:61 sample,

(b) $\mathrm{Ni}(0.01 \mathrm{wt} . \%)$ and

(c) $\mathrm{Ni}(0.5$ wt.\%) doped SBN:61

samples. Dielectric loss $(\tan \delta)$

as a function of temperature at

frequency of $1 \mathrm{kHz}(\mathbf{d})$
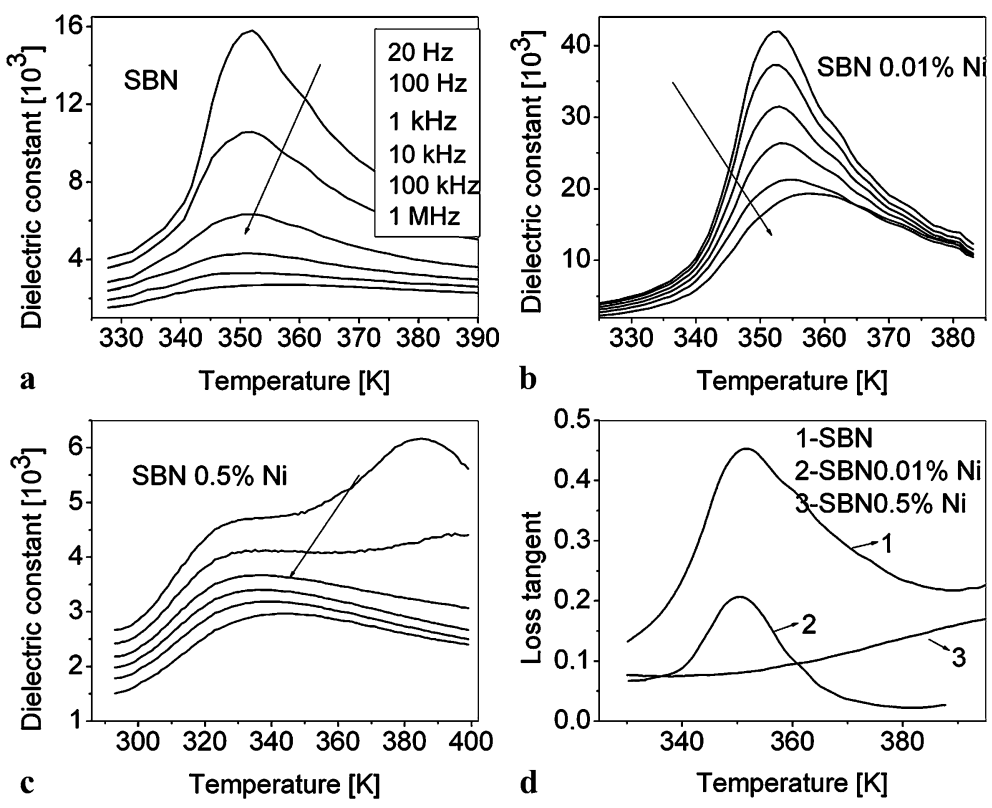

comparison with the undoped one, at the same or even lower applied field. Usually, local defects, dislocations are considered the most probable defects which serve as nucleation centers [29]. The doping leads to generation of additional point defects, which induce a local field, thus promotes domain nucleation. It was reported that dislocation density increased by two orders of magnitude in SBN:61 crystals with dopants of $\mathrm{Ce}$ and $\mathrm{Cr}$ ions, in comparison to undoped samples [31]. It seems also to be reflected in a very fine, irregular domain structure during polarization reversal in the examined Ni doped SBN:61 crystal samples. Moreover, for the slightly doped SBN:61 sample the saturation and remnant polarization increased (about $50 \%$ ), while the $E_{\mathrm{c}}$ decreased by about twice in comparison with the undoped SBN:61 sample, at the same applied ac electric field. Thus, the corresponding increase of dielectric constant $\varepsilon$, as shown in Fig. 6(b), may be connected with the improvement of the switching process in this crystal sample. The nature of a profound effect cannot be analyzed, because the location of $\mathrm{Ni}$ ions in SBN lattice is unknown [9]. It has been suggested that the crystal defects like oxygen vacancies accumulate at the domain boundaries and result in strong domain pinning. It has been found that the oxygen vacancies have a lower formation energy in the domain wall than in the bulk [32]. The introduction of the Ni doping with excessive oxygen atoms may have a strong effect in increasing domain reorientation by reducing the oxygen vacancy density, and consequently the pinning effect on the domain walls. Therefore, the $\mathrm{Ni}$ substitution at a small amount is effective in compensating the chemical and electronic defects, and thereby decreasing (twice) the electric conductivity of slightly doped SBN: 61 sample, and the low substituting level does not affect the temperature $T_{\mathrm{m}}$ characterizing dielectric anomalies.
The introduction of $\mathrm{Ni}$ ions at higher concentration cause an increase of imperfections in the doped SBN:61 crystal. The inhomogeneous distribution of the domain nuclei (Fig. 4) can reflect the nonuniform defect structure distribution within the crystal sample We have shown that slow and fast domain walls coexist, correspond to different local barriers heights. It means that the domain walls have a finite response time to the applied fields, which is the time required for the wall to overcome the local pinning barriers. The distribution of the heights of local pinning barriers, one can account for the very broad maximum of dielectric permittivity, $\varepsilon(\mathrm{T})$ function, and can explain the small values of dielectric permittivity in SBN:61 with a higher Ni concentration, as presented in Fig. 6(c). We have found that the switching process in the highly doped sample requires the field exposure times that can range up to several tenths of seconds, even for $E>E_{\mathrm{c}}$. In consequence, the domain walls in the regions with a more stabilizing domain structure cannot follow the electric field changes, at high frequency of a weak ac-measuring field. For such crystals, low dielectric permittivity values are obtained in comparison with undoped or slightly doped samples, as is shown in Fig. 6.

It was reported that the Curie temperature for a ferroelectric transition depends on the material composition [33, 34]. Our results obtained for highly defected crystals may confirm that the compositional disorder could play an important role on the ferroelectric phase transition diffuseness and the low frequency dielectric response. The crystal compositional disorder, gives rise to the phase transition diffuseness, assuming the existence of microregions that reveal different phase transition temperatures. It was recently confirmed by the PFM technique that the local ferroelectric characteris- 
tics remain well beyond the phase transition temperature in SBN:61 crystals [16].

\section{Conclusions}

The use of the NLC method offers a relatively simple and rapid means of quality control in detecting the distribution of the domain nuclei during the switching process in SBN:61 crystals. The dopants introduced into SBN:61, at low Ni concentration, promote the switching process by reducing the value of threshold nucleation field, and thus coercive field. Such crystals show higher dielectric constant and lower dielectric loss, implying a promising candidate for electrooptics device applications. A significantly broader dielectric anomalies with lower dielectric constant values were observed in undoped and highly doped SBN:61 crystal samples. We speculate that this reflects an internal disorder of both crystal samples, in which one could distinguish regions that exhibit switchable polarization and regions with more fixed polarization.

Open Access This article is distributed under the terms of the Creative Commons Attribution Noncommercial License which permits any noncommercial use, distribution, and reproduction in any medium, provided the original author(s) and source are credited.

\section{References}

1. R.R. Neurgaonkar, J.R. Oliver, W.K. Cory, L.E. Cross, D. Viehlan, Ferroelectrics 160, 265 (1994)

2. E. Soergel, Appl. Phys. B, Lasers Opt. 81, 729 (2005)

3. J. Trull, S. Saltiel, V. Roppo, C. Cojocaru, D. Dumay, W. Krolikowski, D.N. Neshev, R. Vilaseca, K. Staliunas, Y.S. Kivshar, Appl. Phys. B 95, 609 (2009)

4. J. Dec, W. Kleemann, Th. Woike, R. Pankrath, Eur. Phys. J. B 14, $627(2000)$

5. M. Venet, J.S. Guerra, I.A. Santos, J.A. Eiras, D. Garcia, J. Phys., Condens. Matter 19, 026207 (2007)

6. A.M. Glass, J. Appl. Phys. 40, 4699 (1969)

7. W. Kleemann, J. Mater. Sci. 41, 129 (2006)

8. T. Volk, L. Ivleva, P. Lykov, N. Polozkov, V. Salobutin, R. Pankrath, M. Woehlecke, Opt. Mater. 81, 179 (2001)

9. T. Volk, D. Isakov, V. Salobutin, L. Ivleva, P. Lykov, V. Ramzaev, M. Woehlecke, Solid State Commun. 130, 223 (2004)
10. T. Woike, U. Dőrfler, L. Tsankov, G. Weckwerth, D. Wolf, M. Wőhlecke, T. Granzow, R. Pankrath, M. Imlau, W. Kleemann, Appl. Phys. B 72, 661 (2001)

11. I.A. Santos, D.U. Spinola, D. Garcia, J.A. Eiras, J. Appl. Phys. 92, 3251 (2002)

12. T. Volk, V. Salobutin, L. Ivleva, N. Polozkov, R. Pankrath, M. Woehlecke, Phys. Solid State 42, 2129 (2000)

13. M.A. Mohiddon, K.L. Yadav, J. Appl. Phys. 101, 094101 (2007)

14. P. Lehnen, W. Kleemann, Th. Woike, R. Pankrath, Phys. Rev. B 64, 224109 (2001)

15. V.V. Shvartsman, W. Kleemann, T. Łukasiewicz, J. Dec, Phys. Rev. B 77, 054105 (2008)

16. X.Y. Liu, Y.M. Liu, S. Takekawa, K. Kitamura, F.S. Ohuchi, J.Y. Li, J. Appl. Phys. 106, 124106 (2009)

17. R.F. Gainutdinov, T.R. Volk, O.A. Lysova, I.I. Razgonov, A.L. Tolstikhina, L.I. Ivleva, Appl. Phys. B 95, 505 (2009)

18. L. Tian, D.A. Scrymgeour, V. Gopalan, J. Appl. Phys. 97, 114111 (2005)

19. V.Ya. Shur, D.V. Pelegov, V.A. Shikhova, D.K. Kuznetsov, E.V. Nikolaeva, E.L. Rumyantsev, O.V. Yakutova, T. Granzow, Ferroelectrics 374, 33 (2008)

20. N.R. Ivanov, T.R. Volk, L.I. Ivleva, S.M. Chumakova, A.V. Ginsberg, Crystallogr. Rep. 47, 1023 (2002)

21. K. Matyjasek, K. Wolska, S.M. Kaczmarek, R.Z. Rogowski, J. Phys., Condens. Matter 20, 295218 (2008)

22. P.B. Jamieson, S.C. Abrahams, J.L. Bernstein, J. Chem. Phys. 48, 5048 (1968)

23. L.I. Ivleva, N.V. Bogodaev, N.M. Polozkov, V.V. Osiko, Opt. Mater. 4, 168 (1995)

24. N.A. Tikhomirova, L.J. Dontsova, S.A. Pikin, L.A. Shuvalov, JETP Lett. 29, 34 (1979)

25. V.Ya. Shur, J. Mater. Sci. 41, 199 (2006)

26. K. Matyjasek, K. Wolska, R.Z. Rogowski, S.M. Kaczmarek, L.I. Ivleva, Ferroelectrics 413, 311 (2011)

27. V.Ya. Shur, D.K. Kuznetsov, A.I. Lobov, E.V. Nikolaeva, M.A. Dolbilov, A.N. Orlov, V.V. Osipov, Ferroelectrics 341, 85 (2006)

28. R.V. Chamberlin, Phase Transit. 65, 169 (1998)

29. M.E. Lines, A.M. Glass, Principles and Applications of Ferroelectrics and Related Materials (Oxford University Press, Oxford, 2001)

30. J. Fousek, V. Janoušek, Phys. Status Solidi 13, 195 (1966)

31. T. Łukasiewicz, M.A. Swirkowicz, J. Dec, W. Hofman, W. Szyrski, J. Cryst. Growth 310, 1464 (2008)

32. L. He, D. Vanderbilt, Phys. Rev. B 68, 134103 (2003)

33. R.A. Ali, C.L. Wang, M. Yuan, Y.X. Wang, W.L. Zhong, Solid State Commun. 129, 365 (2004)

34. C. Perrin, N. Menguy, O. Bidault, C.Y. Zahra, A.-M. Zahr, C. Caranoni, B. Hilczer, A. Stepanov, J. Phys., Condens. Matter 13, 10231 (2001) 